\title{
NIR fluorescence-guided tumor surgery: new strategies for the use of indocyanine green
}

This article was published in the following Dove Press journal:

International Journal of Nanomedicine

\author{
Claire Egloff-Juras (iD) ${ }^{1,2}$ \\ Lina Bezdetnaya 1,3 \\ Gilles Dolivet ${ }^{1,3}$ \\ Henri-Pierre Lassalle iD 1,3 \\ 'Université de Lorraine, CNRS, CRAN, \\ Nancy F-54000, France; ${ }^{2}$ Université de \\ Lorraine, CHRU-Nancy, Institut de \\ Cancérologie de Lorraine, Nancy F-54000, \\ France; ${ }^{3}$ Institut de Cancérologie de \\ Lorraine, Nancy F-54000, France
}

\begin{abstract}
Surgery is the frontline treatment for a large number of cancers. The objective of these excisional surgeries is the complete removal of the primary tumor with sufficient safety margins. Removal of the entire tumor is essential to improve the chances of a full recovery. To help surgeons achieve this objective, near-infrared fluorescence-guided surgical techniques are of great interest. The concomitant use of fluorescence and indocyanine green (ICG) has proved effective in the identification and characterization of tumors. Moreover, ICG is authorized by the Food and Drug Administration and the European Medicines Agency and is therefore the subject of a large number of studies. ICG is one of the most commonly used fluorophores in near-infrared fluorescence-guided techniques. However, it also has some disadvantages, such as limited photostability, a moderate fluorescence quantum yield, a high plasma protein binding rate, and undesired aggregation in aqueous solution. In addition, ICG does not specifically target tumor cells. One way to exploit the capabilities of ICG while offsetting these drawbacks is to develop high-performance near-infrared nanocomplexes formulated with ICG (with high selectivity for tumors, high tumor-to-background ratios, and minimal toxicity). In this review article, we focus on recent developments in ICG complexation strategies to improve near-infrared fluorescence-guided tumor surgery. We describe targeted and nontargeted ICG nanoparticle models and ICG complexation with targeting agents.
\end{abstract}

Keywords: near-infrared fluorescence-guided surgery, indocyanine green, nanoparticle, targeted nanoparticle, ICG complexation

\section{Introduction}

\section{Fluorescence-guided surgery}

Surgery is the frontline treatment for a large number of cancers. ${ }^{1,2}$ Between $63 \%$ and $98 \%$ of patients with lung, breast, bladder, or colorectal cancer will undergo a surgical resection. ${ }^{3}$ The objective of these excisional curative surgeries is the complete removal of the primary tumor with sufficient safety margins. Obtaining negative margins of excision is essential to improve the chances of a full recovery. Indeed, positive margins can be the cause of local recurrence for $75 \%$ of patients ${ }^{4}$ and the development of distant metastases or a second histologically linked cancer. ${ }^{5-7}$

Intraoperative evaluation of these margins consists of visual inspection and palpation and is followed by extemporaneous histological evaluation, which sometimes takes time and can be inaccurate. ${ }^{5}$ It is not uncommon to find discrepancies between the selective intraoperative evaluation and the more complete evaluation of
Correspondence: Claire Egloff-Juras Faculté d'Odontologie de Lorraine, Université de Lorraine, 7 Avenue de la Forêt de Haye, Vandoeuvre-lès-Nancy, Nancy 54500, France

Tel +33 372746760

Email claire.juras@univ-lorraine.fr 
the operative specimen. Despite the progress made in the various areas of preoperative imaging (computerized tomography, magnetic resonance imaging), the positive surgical margin rate has not significantly decreased in recent years. $^{8}$

However, progress appears possible, for example by using fluorescence-guided surgery (FGS) techniques with fluorescent probes or nanoparticles to detect cancerous tissue in real time. ${ }^{9,10}$ These techniques are noninvasive and have high resolution. ${ }^{11}$ The concept of FGS is well known, as it has been used in neurosurgery since $1948 .^{12}$ Brain tumors remain a major indication for FGS; these tumors are infiltrative and safety margins can never be achieved. $^{13,14}$

In connection with the development and emergence of new technologies, FGS is again the subject of many studies. Most techniques developed for FGS consist of the use of a fluorescence camera with a near-infrared (NIR)sensitive detector. The advantages of NIR light (700$900 \mathrm{~nm}$ ) include its high tissue penetration (up to several centimeters deep) and the low autofluorescence of biological tissues, thus providing sufficient contrast. ${ }^{15}$ In addition, NIR wavelengths are not visible to the human eye and therefore do not change the surgeon's vision of the surgical field. ${ }^{16}$ These advantages have resulted in the development of different imaging systems adapted for guided surgery. ${ }^{17-19}$

\section{Indocyanine green}

The concomitant use of fluorescence and indocyanine green (ICG) has proved effective in the identification and characterization of tumors and metastatic lymph nodes. ${ }^{16,20-23}$ It is authorized by the Food and Drug Administration (FDA) and the European Medicines Agency and is therefore the subject of a large number of studies. Nevertheless, it is important to note that ICG is approved by the FDA for determining cardiac output, hepatic function, liver blood flow, and for ophthalmic angiography, and ICG-based FGS is currently only used in clinical trials. It also has a good signal-to-noise ratio due to low autofluorescence and high tissue penetration at a wavelength of $820 \mathrm{~nm}^{24}$

Before hepatologists, cardiologists, nephrologists, and ophthalmologists used it as a diagnostic tool, ICG was used as a photographic dye from the late 1940s. It was subsequently rediscovered as a fluorophore that absorbed and emitted light in the NIR. ${ }^{25}$ ICG is one of the most common fluorophores used in NIR fluorescence-guided techniques. It is an anionic, amphiphilic, water-soluble fluorophore with a molecular weight of $776 \mathrm{Da}^{26,27}$ It binds rapidly to plasma proteins after being injected intravenously. Its excitation and emission peaks are respectively 780 and $820 \mathrm{~nm}$.

However, ICG also has some disadvantages, including limited photostability, a moderate fluorescence quantum yield, a high plasma protein binding rate, and undesired aggregation in aqueous solution. ${ }^{11}$ The short persistence of fluorescence of the free dye in vivo is attributed to fluorescence quenching of free ICG in physiological environments with rapid aggregation and clearance from the body. ${ }^{28}$ Moreover, ICG is not a specific marker for tumor cells. $^{29}$ An opportunity exists to exploit the features of ICG while offsetting these disadvantages, by designing high-performance NIR nanocomplexes formulated with ICG (with high selectivity for tumors, high tumor-to-background ratios, and minimal toxicity). ${ }^{19,30-35}$ The vectorization of ICG with nanoparticles results in its protection and thus increases its circulation time. The biodistribution and the in vivo availability is thus more interesting. The binding of different ligands, either on ICG nanoparticles or directly on ICG, results in tumor tissue-specific labeling.

In this review article, we have focused on recent developments in ICG complexation strategies to improve NIR FGS.

\section{Indocyanine green nanoparticles Nanoparticles}

The purpose of using nanoparticles or nanovectors is to increase the efficacy, specificity, and biosafety of therapeutic or imaging agents. A nanoparticle is defined as an object sized between 5 and $200 \mathrm{~nm},{ }^{36}$ which is around the size of a protein or a virus.

There are three major generations of nanoparticles. The first generation corresponds to stable colloids in aqueous media encapsulating active agents; the main aim is to slow down their elimination (especially via the renal route) and prolong their effects. Nevertheless, this class of nanoparticles does not escape the immune system.

Second-generation nanoparticles are termed "stealthy", as they have undergone surface modifications allowing them to escape the immune system. ${ }^{37}$ This escape leads to an increase in the nanoparticle plasma half-life. The most commonly used surface treatment is polyethylene glycol (PEG) because it is biocompatible, releasable in 
aqueous media, nontoxic, and weakly immunogenic. This type of nanoparticle allows passive tumor targeting.

Third-generation nanoparticles are stealthy and targeted. Their surface is functionalized with biological ligands recognizing specific targets. The ligands can be extremely varied in nature and allow active tumor targeting.

\section{ICG nanoparticles}

The use of ICG for fluorescence-guided surgical techniques presents a number of constraints; ICG has a low fluorescence quantum yield in solution due to selfquenching ${ }^{38}$ but also a rapid elimination by the liver after intravenous administration. ${ }^{39}$ To overcome this, it is possible to incorporate ICG into nanoparticles and thus improve stability and circulation time and facilitate the accumulation of ICG in tumors. ${ }^{40}$ ICG can be loaded, doped, or conjugated to different types of nanoparticles. These nanoparticles can themselves be bound to ligands and thus allow tumor-specific targeting.

In recent years, there has been a steady increase in the development of nanoparticles for the treatment and imaging of cancers. There are currently 225 clinical trials involving the use of nanoparticles in oncology and two using ICG nanoparticles. However, there is still a limited number of clinically approved nanoparticles.

\section{ICG nanoparticles for passive tumor targeting Enhanced permeability and retention effect}

For tumor imaging, the passive targeting of tumor tissue by nanoparticles relies mainly on the EPR (enhanced permeability and retention) effect. The EPR effect results from poor vascular formation in solid tumors with openings between endothelial cells, resulting in preferential retention of macromolecules and nanoparticles. ${ }^{41-43}$ When tumors reach a sufficient size, oxygen and nutrients cannot diffuse fast enough inside the tumor. ${ }^{44}$ When this happens, growth factors are released that trigger angiogenesis in the surrounding tissue, forming new blood vessels. ${ }^{2}$ However, the vascularization formed is not well structured, which leads to increased extravasation of all molecules that intercalate between discontinuities in the endothelial layer, ranging from 200 to $2000 \mathrm{~nm}$. After extravasation, larger particles are retained preferentially in the tumor because of the deficiency of the lymphatic system. ${ }^{2}$ This is advantageous, as many first-generation nanoparticles are not targeted, and this phenomenon is therefore used to allow the release of therapeutic compounds passively within solid tumors.

\section{Passive tumor targeting}

Passive targeting requires the use of stable, nonaggregated, stealthy compounds that have a sufficiently long circulation time to result in accumulation in tumors. It also requires that the compounds are of a size that does not exceed the pore size of permeabilized vessels (between 380 and $780 \mathrm{~nm})^{45,46}$ and which is not too small. The EPR effect is negligible for small molecules that can be redistributed into the bloodstream by diffusion and/or convection and are eliminated preferentially by the renal route. ${ }^{47}$

However, because of the variability in the structure and extent of the vascular system, tumor stroma, and lymphatic drainage, the EPR effect varies depending on the type and location of the tumor, making it a useful but not universal method of targeting. For example, it is more prominent in small tumors, which is probably related to a higher density of vessels compared with large tumors with an avascular region. $^{48}$

It is interesting to note that the tumor targeting that exists during the injection of ICG alone is also explained by the EPR effect, due to the fact that upon administration, ICG binds to serum proteins.

\section{Different types of ICG nanoparticles Inorganic ICG nanoparticles}

The characteristics (nature, size) of the nanoparticles described in this section and the tumor models used are presented in Table 1.

One of the first types of ICG inorganic nanoparticles developed was the mesoporous silica nanoparticle. In 2009 , Lee et $\mathrm{al}^{49}$ were interested in the characterization and the in vivo biodistribution of mesoporous silica nanoparticles loaded with ICG for optical imaging and demonstrated their effectiveness. They found a reduction in ICG aggregation, fluorescence self-quenching, and host immune response. This type of nanoparticle has the advantage of an excellent biocompatibility and easy functionalization with different compounds. ${ }^{50}$

The same team was subsequently interested in the impact of the surface charge of mesoporous silica nanoparticles on in vivo biodistribution and found that positively charged nanoparticles were excreted from the liver into the gastrointestinal tract much faster than negatively 


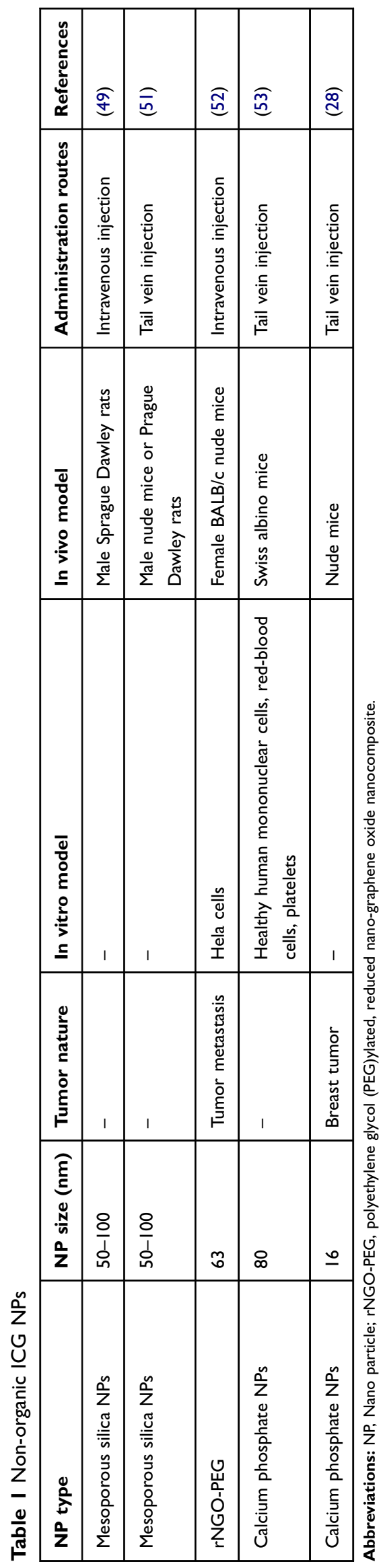

charged nanoparticles. ${ }^{51}$ The authors concluded that excretion and clearance are influenced by surface charge and that positively charged mesoporous silica nanoparticles are more easily eliminated than negatively charged ones.

Chen et al designed an ICG-loaded, PEGylated, reduced nano-graphene oxide nanocomposite (rNGOPEG/ICG) for photoacoustic and fluorescence dual-modality imaging. ${ }^{52}$ They reported an excellent fluorescence signal both in vitro and in vivo, with minimal toxicity. These nanoparticles possessed greater stability, a longer blood circulation time, and superior tumor targeting capability compared with free ICG. In mice, rNGO-PEG/ICG was found to circulate for up to $6 \mathrm{hrs}$ after intravenous injection, and after 1 day no accumulation of nanoparticles was found in any major organs except the tumor regions. The authors concluded that their rNGO-PEG/ICG nanocomposite is capable of effectively targeting tumors in vivo via the EPR effect.

ICG-encapsulating calcium phosphate nanoparticles have also been developed, ${ }^{28,53}$ and have improved ICG loading efficiency and quantum yield compared to polymeric nanoparticles. ${ }^{28}$ Calcium phosphate nanoparticles can be used for in vivo tumor imaging and also for drug, gene, or siRNA delivery, with the huge advantage of being nontoxic. ${ }^{53}$ ICG encapsulation significantly enhanced emission intensity compared to free ICG at elevated concentrations. ${ }^{40}$ Altinoglu et al developed PEGylated calcium phosphate nanoparticles with a prolonged circulation time in vivo. ${ }^{28}$

In addition, Song et $\mathrm{al}^{54}$ developed small ICG-loaded magnetic carbon nanoparticles (MCNPs) for combined fluorescent and MRI imaging, which efficiently accumulated in tumors due to the EPR effect and the small particle size.

\section{In vivo biodistribution of inorganic nanoparticles}

In mouse model studies, in vivo biodistribution of inorganic nanoparticles demonstrated a strong and stable fluorescence, prominent in the liver. ${ }^{49,51}$ In one study, there was a strong accumulation in internal organs such as the liver and intestine from 0.5 to $6 \mathrm{hrs}$. Over time, accumulation of the nanoparticles toward the tumor region became more significant and after $48 \mathrm{hrs}$ the fluorescence signals were found only in the tumor region. ${ }^{52}$ In contrast, the clearance process for free ICG was rather rapid and the majority of the dye was cleared out of the body after $6 \mathrm{hrs}^{34}$

In conclusion, the fluorescence signals from ICG nanoparticles are significantly extended in vivo compared to free ICG. 


\section{Organic ICG nanoparticles}

The characteristics (nature, size) of the nanoparticles described in this section and the tumor models used are presented in Table 2. Poly(lactic-co-glycolic acid) (PLGA) carriers are one of the most common biodegradable and biocompatible systems used for ICG loading. ${ }^{40}$ Zhao et al developed ICG-loaded PLGA-lecithin-PEG core-shell nanoparticles of $39 \mathrm{~nm}, 68 \mathrm{~nm}$, or $116 \mathrm{~nm}$ in size via single-step nanoprecipitation. ${ }^{55}$ The aim of their study was to explore the effects of nanoparticle size on biodistribution and tumor accumulation. They compared the excretion time of free ICG versus that of their nanoparticle. Fluorescence imaging indicated that nanoparticles were retained within the body longer than free ICG, which was quickly excreted and cleared. They also concluded there was a size-dependent tumor accumulation of ICG-PLGA nanoparticles: the $68 \mathrm{~nm}$ particles could easily pass through the vessel pores and had slower clearance than $39 \mathrm{~nm}$ particles.

$\mathrm{Wu}$ et al developed a nanoparticle in which ICG was encapsulated in the core of a polymeric micelle, which selfassembled from amphiphilic PEG-polypeptide hybrid triblock copolymers of poly(ethylene glycol)-b-poly(L-lysine)-b-poly (L-leucine) (PEG-PLL-PLLeu), with PLLeu as the hydrophobic core and PEG as the hydrophilic shell. ${ }^{56}$ The ICG was associated with the hydrophobic core via hydrophobic interaction and with the hydrophilic heads through electrostatic attractive interaction. Compared with free ICG, PEG-PLLPLLeu-ICG micelles significantly improved quantum yield and fluorescent stability. The cellular uptake experiments showed that PEG-PLL-PLLeu-ICG micelles had a high cellular uptake rate. In addition, in vivo experiments revealed an excellent passive tumor targeting ability and long circulation time.

This and other studies ${ }^{57-59}$ demonstrates that the encapsulation of ICG into polymer-based nanoparticles positively influences the properties of ICG.

Liposomes are another type nanoparticle frequently used for encapsulating ICG. Liposomes are closed spherical vesicles composed of a lipid bilayer made of either synthetic or natural phospholipids and have diameters of about $100 \mathrm{~nm}$. Because ICG is an amphiphilic dye, it can be loaded either inside the lipid core or at the interface of lipid nanoparticles. Liposomes can protect the inside agents from the surrounding environment and prolong circulation time in the body. Another advantage is that liposomal ICG can be made entirely of clinically approved components. ${ }^{40,60}$

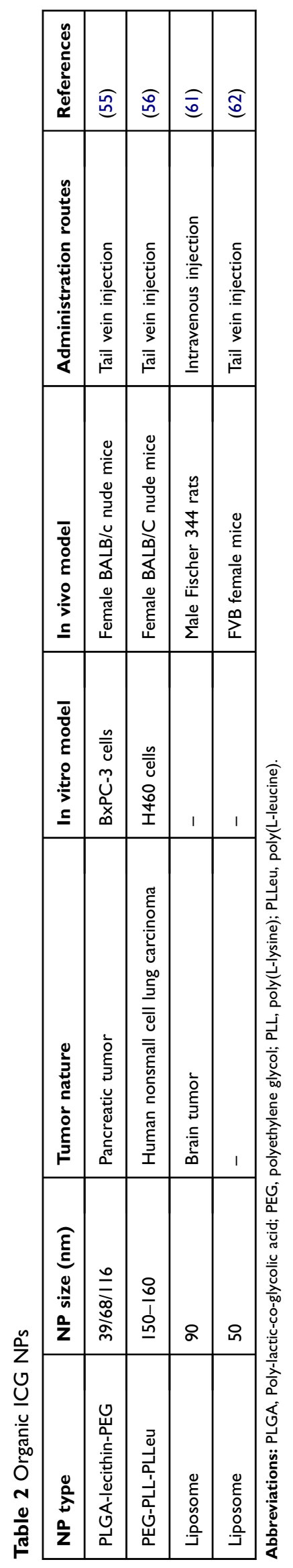


Suganami et al explored the use of a liposomally formulated phospholipid-conjugated ICG for intraoperative brain tumor detection, ${ }^{61}$ and confirmed that this nanoparticle allowed the real-time detection of tumor and normal brain tissues.

In the study of Mérian et al, liposomal forms of ICG showed similar biodistribution patterns as free ICG, which indicate the immediate leakage from the nanoparticle after intravenous injection. ${ }^{62}$ They also demonstrated that ICG leakage increased considerably in the presence of bovine serum albumin because of the high affinity of the fluorophore for plasma proteins.

\section{In vivo biodistribution of organic nanoparticles}

Organic nanoparticles have excellent passive tumor targeting ability and a long circulation time. They are released slowly, which avoids the strong binding of ICG to nonspecific plasma proteins and the subsequent rapid elimination from the body (a half-life of 3-4 min). In mice treated with free ICG, fluorescence appeared in the liver and kidneys 4 hrs after injection, showing a dominant uptake of ICG by these organs. Twenty-four hours after injection, no detectable signal was recorded from free ICG, in contrast to what is observed with ICG nanoparticles. ${ }^{56}$

Zhao et al demonstrated that the in vivo biodistribution varies according to the size of the nanoparticles. Size could not only influence endocytosis but also affect tumor accumulation. ${ }^{55}$

The architecture of these inorganic and organic nanoparticles allowed ICG protection and so enhanced biocompatibility, photostability, and tumor accumulation by increasing the EPR effect. Nevertheless, the specificity of tumor labeling with ICG by passive targeting remains limited as previously described. This limitation explains the growing development of formulations allowing active tumor targeting.

\section{ICG nanoparticles for active tumor targeting}

Active tumor targeting by nanoparticles loaded with ICG is based on two elements: the target and the molecule recognizing the target, which must be grafted onto the nanoparticle. The target must be carefully chosen. It must be accessible (expressed on the surface of cells) and specific to the tumor (or at least be expressed more strongly in tumors). There must also be a ligand with high selectivity and high affinity for the target.

\section{Folic acid-conjugated nanoparticles}

Folate receptors are highly overexpressed on the surface of many tumor types. This makes folic acid (FA) a prime ligand for the active targeting of tumor tissues. Table 3 summarizes reported types of FA-conjugated nanoparticles with their characteristics (type, size) and the chosen tumor model.

One of the most common forms of ICG-loaded nanoparticles coupled with FA is a polymer and liposome assembly (lipid-polymer hybrid nanoparticles, which have a biodegradable polymeric core surrounded by a lipid monolayer). These lipid-polymer hybrid nanoparticles have the combined advantages of polymeric nanoparticles and liposomes. ${ }^{63}$ Chopra $^{63}$ developed FA-conjugated PLGA-lipid nanoparticles containing ICG at the core, called FA-ICG-PLGA-lipid nanoparticles. The imaging properties and biodistribution of FA-ICGPLGA-lipid nanoparticles have been compared to those of free ICG and of the nanoparticles without FA. Twenty-four hours after injection, only the fluorescence signal of FA-ICGPLGA-lipid nanoparticles could be detected in tumors. NIR fluorescence imaging of the various organs and tumors ex vivo demonstrated significant differences between the three compounds tested, with the best tumor fluorescence obtained with FA-ICG-PLGA-lipid nanoparticles. Xin et $\mathrm{al}^{64}$ synthesized PLGA-lipid nanoparticles (PNPs) that covalently conjugate FA and ICG, called FA-RIPNPs. They concluded that FARIPNPs may facilitate a high cellular uptake via folate receptor-mediated endocytosis and that these nanoparticles have excellent stability and biocompatibility characteristics. FARIPNPs exhibited a 2.8-fold higher tumor accumulation than RIPNPs without FA, and a 12.6-fold higher accumulation than free ICG. The authors also added a PEG shell around the PLGA core to prolong the circulation time. Zheng et $\mathrm{al}^{65}$ also described the same type of nanoparticles. These were ICG dye-doped PLGA-lipid folate receptor-targeted nanoparticles (FA-ICG-PLGA-lipid nanoparticles) constructed by employing a single-step self-assembly and nanoprecipitation method. The FA-ICG-PLGA-lipid nanoparticles exhibited excellent optical properties compared to free ICG (high aqueous stability, significant stability against photobleaching, excellent NIR optical properties), highly selective tumor localization, and prolonged circulation time in vivo. In the same way, Zheng et al ${ }^{66}$ developed an ICG-containing nanostructure utilizing the noncovalent self-assembly chemistry between phospholipid-PEG (PL-PEG) and ICG. They used two different cellular models: U87 cancer cells, which overexpress the integrin $\operatorname{Rv} \beta 3$ on the cell surface, and MCF-7 


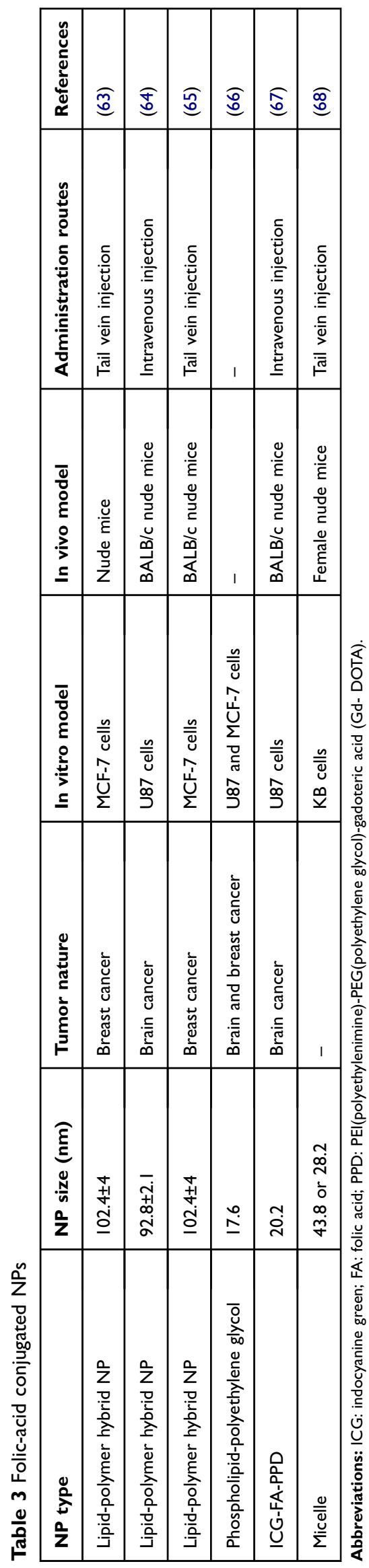

cancer cells, which express a very low level of integrin $\operatorname{Rv} \beta 3$. These models were used because the nanoparticles were functionalized both with FA and with monoclonal antibodies (mAbs) to Rvß3.

Another possible design is that proposed by Wu et al ${ }^{67}$ a multifunctional nanostructure (ICG-FA-PPD), which consisted of the self-assembly of ICG- and FA-modified PEI (polyethylenimine)-PEG-gadoteric acid (FA-PPD). This nanoparticle showed greater photostability and lower cytotoxicity than free ICG and significantly accumulated in the tumor.

For ICG-loaded nanoparticles functionalized with FA, micelles could also be used. For example, Yan and Qui ${ }^{68}$ encapsulated ICG into folate-conjugated poly(2-ethyl-2oxazoline)-b-poly( $\varepsilon$-caprolactone) micelles. In this nanoparticle, the poly(2-ethyl-2-oxazoline) plays the "stealth" role to extend blood circulation and suppress unwanted uptake by liver and spleen and the poly( $\varepsilon$-caprolactone) serves as drug storage to improve the stability of ICG. With their configuration, the authors obtained a significantly higher ICG stability compared to free ICG in vitro and in vivo and an excellent biocompatibility. These micelles could effectively target tumors and realize longterm tumor imaging with high contrast resolution. Also of interest is that the fluorescence of the ICG-loaded micelles was stable at room temperature for 14 days. ${ }^{69}$

\section{Hyaluronic acid-conjugated nanoparticles}

Several studies have coupled ICG nanoparticles with hyaluronic acid. This allows both passive targeting (by the EPR effect due to nanoparticles) and active targeting (due to the affinity of hyaluronic acid for CD44). Uthaman et $\mathrm{al}^{70}$ hydrophobically modified ICG prior to loading it into hyaluronic acid-based micelles and utilized these nanoparticles for CD44-based targeting. They used hyaluronic acid-C18 (octadecylamine) micelles, explaining that this type of micelle is better than the systems in which ICG is conjugated on the polymer. They observed protection of ICG by micelles from degradation and clearance until complete imaging.

In the same way, Qi et al ${ }^{71}$ utilized hyaluronic acidderived nanoparticles with physico-chemically entrapped ICG, termed NanoICG, for intraoperative NIR fluorescence of pancreatic cancer. This formulation improved the poor aqueous solubility of imaging agents. ${ }^{72}$ NanoICG accumulated significantly within the pancreas in an orthotopic pancreatic adenocarcinoma model and demonstrated contrast enhancement for pancreatic lesions 
relative to nondiseased portions of the pancreas: ICG accumulation in the tumor was increased compared with free ICG and the fluorescence intensity of NanoICG was higher compared with ICG both in pancreas and splenic metastases. The in vitro and in vivo experimentation indicated negligible toxicity of NanoICG.

However, a limitation of hyaluronic acid-conjugated nanoparticles is their preferential uptake by the reticuloendothelial system, ${ }^{72}$ probably due to their high molecular weight. ${ }^{71}$

\section{Antibody-conjugated nanoparticles}

ICG-loaded nanoparticles can also be conjugated with antibodies. Table 4 describes the characteristics (nature, size, ligand/target) of antibody-conjugated nanoparticles and the tumor models used.

mAbs against the human epidermal growth factor receptor-2 (HER-2) is of great interest for imaging of active tumor targeting. ${ }^{73-75}$ HER-2 is involved in the carcinogenesis of various solid tumors, such as breast, colorectal, nonsmall cell lung, and ovarian cancer. ${ }^{76,77}$ Different designs have been investigated, including nanocapsules, ${ }^{73}$ erythrocytederived transducers, ${ }^{74}$ and silica nanoparticles. ${ }^{75}$ All these designs resulted in a significantly higher fluorescence in cells overexpressing HER-2 compared with cancer cells expressing lower HER-2 levels; the study authors concluded that successful tumor targeting is achieved by conjugating nanoparticles with anti-HER-2 antibodies.

Chen et $\mathrm{al}^{78}$ proposed anti-NGAL (neutrophil gelatinase-associated lipocalin)-conjugated gold nanoshells that specifically targeted pancreatic cells in vitro and in vivo. They designed a theranostic nanocomplex targeted to NGAL and observed a higher tumor contrast compared with nontargeted PEGylated nanoparticles.

To target colorectal adenocarcinoma, Kolitz-Domb et al ${ }^{79}$ synthesized a proteinoid-poly(L-lactic acid) copolymer bound to an anti-carcinoembryonic antigen (anti-CEA) antibody. The encapsulation of the ICG within this nanoparticle improved its photostability (by avoiding leakage and photobleaching) and targeting with anti-CEA antibodies achieved specific colon tumor detection in vivo.

Finally, using ICG-containing PL-PEG coupled with anti-Rvß3 mAbs, Zheng et $\mathrm{al}^{66}$ observed a higher ICG fluorescence intensity in cells overexpressing the integrin $\operatorname{Rv} \beta 3$ compared to cell lines with few cell surface receptors.

\section{Nanoparticles coupled with other ligands}

To target prostate cancer cells (PC3), PLGA nanoparticles loaded with ICG and conjugated with RGD-4C (specific to

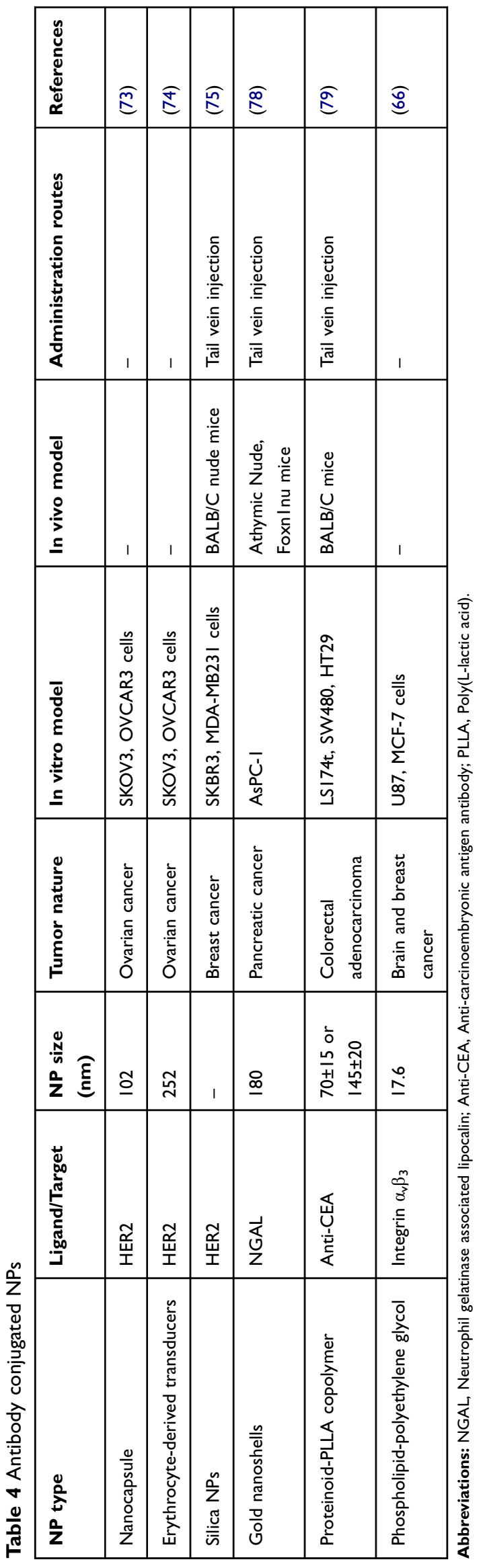


$\alpha 5 \beta 3$ ) or R11 peptides can be used. ${ }^{80}$ R11 peptides are cell-penetrating peptides specific to prostate cancer cells. In vitro studies showed that these nanoparticles had an improved optical stability (compared with free ICG), excellent biocompatibility with healthy cells, and an effective targeting capability.

Liposome-encapsulated ICG can also be coupled with various ligands, such as the RGD (Arg-Gly-Asp) peptide used to target gastric cancer. ${ }^{81}$ This formulation had a higher stability than free ICG, showed greater accumulation in the tumor tissues, and exhibited prolonged circulation time leading to an improved in vivo visualization of tumor tissues.

\section{Cancer cell membrane-coated nanoparticles}

Chen et $\mathrm{al}^{82}$ proposed another way of achieving active tumor targeting with ICG-loaded nanoparticles: using cancer cell membrane-cloaked nanoparticles. They synthesized biomimetic nanoparticles called ICNP (for "ICG-loaded and cancer cell membrane-coated nanoparticles"), which have a core-shell nanostructure consisting of an ICG-polymeric core and a cancer cell membrane shell (Figure 1). Due to their membrane structure and antigens, this type of active cell membrane-cloaked nanoparticle allows specific tumor binding, a long blood circulation time, and immune system escape. Nude mice bearing MCF-7 tumors received tailvein injections of free ICG, nanoparticles without a cancer cell membrane coating, or ICNPs. ICNPs significantly promoted cell endocytosis and homologous-targeting tumor accumulation in vivo. These nanoparticles possessed both passive and homologous active targeting with a significantly enhanced tumor accumulation 24 hrs after injection. Nevertheless, these nanoparticles only target the type of tumor from which the membrane that covers them originates. It is therefore necessary to obtain nanoparticles for each tumor type.

\section{In vivo biodistribution of targeted nanoparticles}

Strong fluorescence signals were obtained in the whole body 5 mins after injection of free ICG, nanoparticles, and targeted nanoparticles. Six hours after injection, however, more intense fluorescence signals were obtained in the tumor region for the targeted nanoparticle group compared with the ICG and nontargeted nanoparticle groups. Twentyfour hours after injection, no detectable fluorescence was recorded in the free ICG and nontargeted nanoparticles groups. In contrast, the tumors of the targeted nanoparticle group exhibited a significant fluorescence signal. ${ }^{64,65}$

The location and tumor margin could be observed $1 \mathrm{hr}$ after injection in mice receiving targeted nanoparticles, but

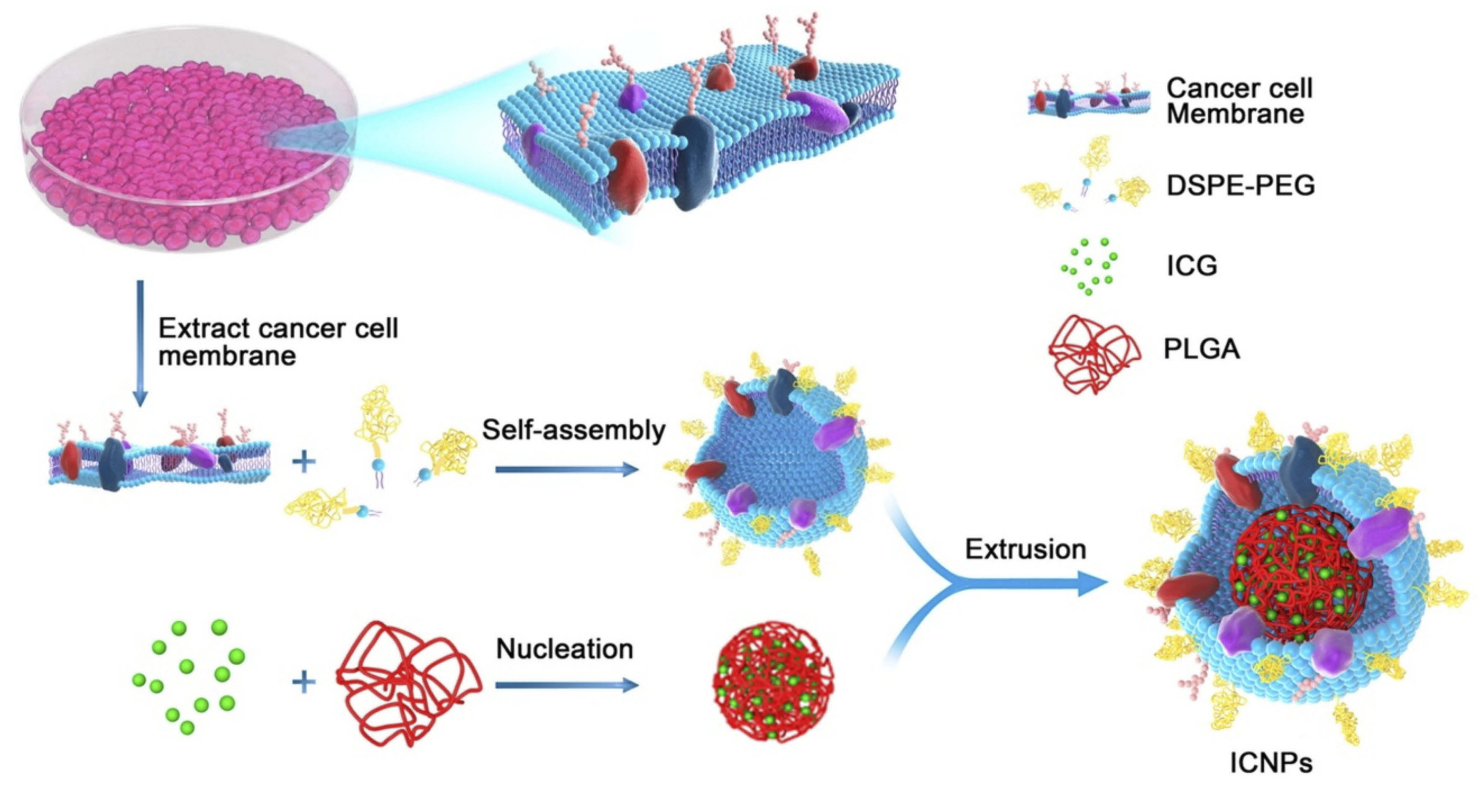

Figure I Illustration of the cancer cell membrane coated nanoparticles with ICG (ICNPs). Preparation procedure of ICNPs: extracting cancer MCF-7 cell membrane hybridized with PEGylated phospholipids (DSPE-PEG) and then coated onto ICG-loaded polymeric cores by extrusion.

Notes: Reprinted with permission from Chen Z, Zhao P, Luo Z, et al. Cancer Cell Membrane-Biomimetic Nanoparticles for Homologous-Targeting Dual-Modal Imaging and Photothermal Therapy. ACS Nano. 2016;10(II):10049-10057. ${ }^{82}$ Copyright () 2016. American Chemical Society. 
not in mice receiving nontargeted nanoparticles or free ICG. $^{68}$

With targeted nanoparticles, the fluorescence gradually diminished but remained detectable for 7 days.

\section{Indocyanine green complexation}

To specifically address a molecule through a mechanism of active targeting, biological conjugation with cancer-specific ligands (such chemical molecules, peptides, proteins, antibodies, and aptamers) is possible. ${ }^{18}$ The binding of cyanine dyes (such as ICG) to nucleic acids or proteins resulted in rigidization of the fluorophores, which increased fluorescence efficiency. ${ }^{83,84}$

\section{ICG conjugated to antibodies}

Many authors report successful conjugation of ICG with antibodies. Table 5 summarizes the different designs proposed as well as the main characteristics (type, size, ligand/target) and the chosen tumor model. The binding of ICG to antibodies allows the creation of activatable NIR probes. ICG loses its fluorescence after protein binding; after cell binding and internalization of ICG-antibody complex, ICG dissociates from the targeting antibody and the fluorescence is therefore activated. ${ }^{27}$ This is related to a unique property of ICG: it loses fluorescence when bound to the antibody, but regains it once it is chemically separated from the antibody, which occurs after internalization and degradation of the conjugate. ${ }^{85}$

A frequently used antibody is the anti-epidermal growth factor receptor (EGFR) $\mathrm{mAb}$, also called panitumumab. ${ }^{27,86-88}$ For antibody conjugation, ICG-sulfo-OSu (ICG-sOSu), an ICG derivative, is often used. ${ }^{85,86}$ It seems that the interaction between ICG-sOSu and panitumumab is due to both covalent and noncovalent binding of the ICG-sOSu to the protein. ${ }^{88}$ Zhou et a ${ }^{88}$ purified the bioconjugate by size-exclusion highperformance liquid chromatography analysis (SE-HPLC), to remove aggregates and impurities, and then removed the noncovalently bound dye by extraction with ethyl acetate to further purify the conjugates. This technique allowed an excellent target-specific uptake of the doubly purified conjugates, with minimal liver retention in vivo.

Sano et $\mathrm{al}^{86}$ re-engineered a panitumumab-ICG conjugate using bifunctional ICG derivatives (ICG-PEG4-SulfoOsu and ICG-PEG8-Sulfo-Osu) with short PEG linkers. PEG has a well-known biocompatibility and is widely used in clinical products. ${ }^{88-90}$ ICG conjugates with PEG linkers significantly increased the percentage of covalent binding of ICG to the antibody, resulting in less in vivo

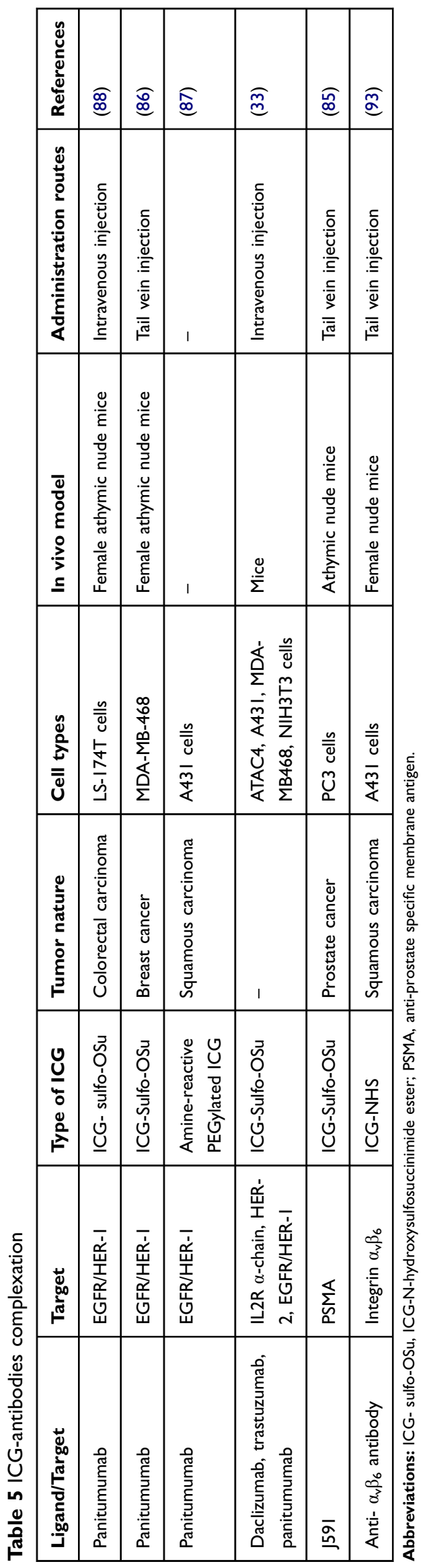


noncovalent dissociation. Compared with the original panitumumab-ICG conjugate without short PEG linkers, the conjugates with PEG linkers were better able to specifically depict EGFR-positive tumors with high tumor-to-background ratios, especially for lesions in or near the liver. PEG linkers also result in a higher stability in serum and a higher in vitro cellular uptake. Villaraza et $\mathrm{al}^{87}$ proposed a similar approach of using PEGylated panitumumab-ICG complexes. For this, a water-soluble amine-reactive PEGylated analogue of $\mathrm{ICG}^{91}$ was synthesized and used to label the panitumumab and was compared with the nonPEGylated conjugate. The PEGylation prevented aggregation in solution, whereas the targeting moiety was conserved. The panitumumab-ICG conjugate has also been used as a photoacoustic imaging probe to target cancerassociated EGFR. ${ }^{92}$

Ogawa et $\mathrm{al}^{27}$ studied three FDA-approved antibodies: daclizumab, trastuzumab, and panitumumab. For each, they successfully characterized the in vivo tumor overexpression of cell surface receptors using their respective ICG-conjugated antibodies. All conjugates demonstrated a high quenching capacity and were activatable, "switching on" only in target cells.

Nakajima et al $^{85}$ designed a humanized anti-prostatespecific membrane antigen (PSMA) antibody (J591) coupled with ICG. They obtained an activatable NIR probe that was able to detect PSMA+ tumors with high contrast compared to PSMA- tumors up to 10 days after injection of a low dose of reagent. Its ability to be activated only in PSMA+ cells led to very high tumor-tobackground ratio.

Finally, Zhang et $\mathrm{al}^{93}$ fabricated an anti-integrin $\alpha \mathrm{v} \beta 6$ antibody and labeled it with ICG to form an ICG- $\alpha v \beta 6$ antibody. The expression of $\alpha \mathrm{v} \beta 6$ integrin is significantly upregulated in cutaneous squamous cell carcinoma (cSCC). ${ }^{94,95}$ This ICG- $\alpha v \beta 6$ antibody complex could detect $\mathrm{cSCC}$ with high specificity and deep penetration $(1 \mathrm{~cm})$.

\section{ICG conjugated to antibody fragments}

Antibody fragments, including diabodies, have more desirable pharmacokinetic characteristics than whole antibodies. For this reason, Sano et $\mathrm{al}^{96}$ proposed an activatable optical imaging probe composed of a PSMA-targeted cysdiabody conjugated with ICG. This complex is only activated when bound to the tumor, which leads to high signal-to-background ratios. For this purpose, they used short PEG linkers between ICG and the reactive functional group, resulting in covalent conjugation of ICG to the cysdiabody. This configuration allowed a lower dissociation of ICG from the cys-diabody after injection and therefore reduced hepatic uptake. However, the authors concluded that whole ICG-conjugated antibodies appeared to be more effective activatable probes for PSMA-specific cancer detection than ICG-conjugated diabody probes. In fact, when ICG is conjugated to small proteins such as diabodies (which are catabolized in the kidneys) it can be recirculated after liver excretion, leading to a prolonged increased background in the abdomen in fluorescence images.

\section{ICG conjugation with other ligands}

ICG can also be conjugated to chlorotoxin. ${ }^{97}$ An aminereactive ester form of ICG was conjugated to chlorotoxin using standard amine-reactive chemistry; the conjugate was termed BLZ-100. The chlorotoxin is derived from the venom of the Leirus quinquestriatus scorpion and presents binding properties in gliomas ${ }^{98}$ and many different solid tumors. ${ }^{99,100}$ The target of the chlorotoxin is not yet known but is thought to involve the membrane proteins annexin $\mathrm{A} 2^{101}$ and metalloproteinase-2. ${ }^{102}$ Baik et $\mathrm{al}^{97}$ aimed to explore the uptake of BLZ-100 in an in vivo model of head and neck squamous cell carcinoma (HNSCC) and to test the ability of BLZ-100 to discriminate among stages of oral dysplasia. The signal-to-background ratio of BLZ-100 was significantly greater than that of free ICG in different HNSCC tumor models. Moreover, BLZ-100 demonstrated highly sensitive and specific uptake at the tumor periphery. This is interesting from a surgical point of view, because the difficulty in operating is not identifying the core of the tumor, but rather identifying the tumor margins and peripheral residual clusters. ${ }^{97}$ BLZ-100 signal increased with dysplasia severity, allowing differentiation between high-risk and low-risk dysplasia.

Transferrin is another ligand that has been used to specifically target ICG to tumor tissue. ${ }^{103}$ A simple mixing of transferrin with ICG enabled their self-assembly in aqueous solution to form nanoparticles that had excellent active targeting abilities and water solubility, colloidal stability, and favorable biocompatibility. There was a high reproducibility because of the simple synthesis procedure. These nanoparticles exhibited an impressive active targeting activity in vitro and in vivo in a cervical carcinoma model. 


\section{Future developments}

ICG nanoparticles for cancer theranostics

Recently, the development of multifunctional nanoparticles offering both diagnostic and therapeutic capabilities has attracted attention. ${ }^{40}$ It is interesting to note that many of the nanoparticles described in this review are multi-modal and exhibit different optical or theranostic properties. For example, the nanoparticles proposed by Chen et $\mathrm{al}^{52}$ enable both photoacoustic and fluorescence tumor imaging. Ashokan et $\mathrm{al}^{53}$ designed a nanoparticle to combine nuclear, magnetic, and NIR in vivo imaging. Nanoparticles can also have drug delivery functions ${ }^{64}$ or photothermal/photodynamic activities. ${ }^{55,56,66,68,73,78}$ Nanoparticles have been reported to cause substantial tumor cell death. This is possibly because ICG can be used as a photosensitizer to generate reactive oxygen species, such as singlet oxygen and superoxide, to damage cancer cells by illumination. ${ }^{40}$

The use of theranostic ICG nanoparticles is of great interest in the management of brain tumors. Fluorescenceguided brain tumor surgery is routinely used in some centers and is under further investigation in clinical trials. ${ }^{104}$ The difficulty of this type of surgery is that it is frequently impossible to resect the entire tumor. In this case, the use of theranostic nanoparticles would allow cellular targeting and thus permit the destruction of all the tumor cells while limiting the associated functional losses.

\section{Second NIR window}

Fluorescent imaging of biological systems in the second NIR window (NIR-II) can probe tissue at centimeter depths and achieve micrometer-scale resolution at millimeter depths. Unfortunately, all current NIR-II fluorophores are excreted slowly and are largely retained within the reticuloendothelial system, making clinical translation nearly impossible. ${ }^{105}$ Currently, the use of ICG as an NIR-II probe is under investigation. ${ }^{106}$

For example, Zeh et al developed a novel way to administer ICG in gliobastoma surgery. This technique, named Second Window ICG, exploits the natural permeability of tumor vasculature and its poor clearance to deliver high doses of ICG to tumors. They obtained an NIR optical contrast with good signal characteristics in intracranial brain tumors over a longer period of time than previously obtained with ICG video angiography alone. This nonspecific tumor accumulation of ICG within the tumor provided strong signal-to-background contrast and was not significantly time dependent between $6 \mathrm{hrs}$ to $48 \mathrm{hrs}$, providing a broad plateau for stable visualization. ${ }^{107}$

\section{Conclusion}

NIR FGS is an innovative and interesting technique for the real-time visualization of resection margins. Accurate visualization of margins would result in decreased risk of recurrence and reduced tissue loss, and would allow detection of tumor micro-foci. ICG, an FDA-approved dye, has been the subject of a large number of studies and is currently the fluorophore of choice for FGS. In this review, we have discussed a large number of ICG complexation strategies. All have the same goal: to overcome the limitations of free ICG, such as poor and exclusively passive tumor targeting, rapid elimination after intravenous injection, and a low fluorescence quantum yield in solution due to self-quenching. Studies have shown that the incorporation of ICG into nanoparticles can increase circulation time, thus increasing the EPR effect and as such passive tumor targeting. Subsequently, the complexation of ICGloaded nanoparticles, or of ICG with different ligands directly, improved the circulation times as well as the optical properties of ICG, achieving active tumor targeting.

Nevertheless, given the absence of systematic studies and the fact that they have been implemented under different conditions, comparison between the various systems is very difficult. Similarly, the large number of different formulations makes their comparison and choosing between them extremely complex.

Considering the number of publications and their year of publication, it seems that future progress is ultimately related to the development of actively targeted nanoparticles loaded with ICG. Only four clinical trials using targeted ICG-conjugated nanoparticles are referenced; nevertheless, these are particularly recent studies (according to the U.S National Library of Medicine). Such nanoplatforms combine the protective benefits of ICG and the immune system escape offered by nanoparticles with the benefits of active targeting enabled by the attachment of different ligands. In addition, there is a greater risk of altering the optical properties of ICG when it is bound directly to ligands than when it is included in nanoparticles. Finally, given the developments in nanotechnology, the different techniques for producing nanoparticles have already been optimized. 
The ultimate aim of all these devices is to be used in the clinical settings. ICG is already FDA approved, and most FDA-approved nanoparticles in oncology are organic nanoparticles. However, of the 55 trials involving nanoparticles, only two clinical trials using ICG nanoparticles were open to inclusion at the end of 2018 (according to the U.S National Library of Medicine). In view of the large number of recent formulations of ICG nanoparticles tested in preclinical models, it is not surprising that for the moment their use in clinical trials is still limited but will undoubtedly progress. One of the main challenges to this transition to the clinic will be to overcome concerns about nanoparticles. In this context, the development of ICG directly conjugated with a targeting agent could, despite some drawbacks, constitute an interesting alternative to ICG-conjugated nanoparticles.

\section{Acknowledgment}

This work was supported by the French "Ligue Nationale Contre le Cancer" and the Institut de Cancérologie de Lorraine.

\section{Disclosure}

The authors report no conflicts of interest in this work.

\section{References}

1. Nagaya T, Nakamura YA, Choyke PL, Kobayashi H. Fluorescenceguided surgery. Front Oncol. 2017;7:314. doi:10.3389/fonc.2017. 00314

2. Hill TK, Mohs AM. Image-guided tumor surgery: will there be a role for fluorescent nanoparticles? Wiley Interdiscip Rev Nanomed Nanobiotechnol. 2016;8:498-511. doi:10.1002/wnan.1381

3. Miller KD, Siegel RL, Lin CC, et al. Cancer treatment and survivorship statistics, 2016. CA Cancer J Clin. 2016;66:271-289. doi:10.33 22/caac. 21349

4. Jones AS, Bin Hanafi Z, Nadapalan V, Roland NJ, Kinsella A, Helliwell TR. Do positive resection margins after ablative surgery for head and neck cancer adversely affect prognosis? A study of 352 patients with recurrent carcinoma following radiotherapy treated by salvage surgery. Br J Cancer. 1996;74:128-132. doi:10.1038/bjc.1996. 327

5. Atallah I, Milet C, Henry M, et al. Near-infrared fluorescence imagingguided surgery improves recurrence-free survival rate in novel orthotopic animal model of head and neck squamous cell carcinoma. Head Neck. 2016;38 Suppl 1:E246-E255. doi:10.1002/hed.23980

6. Clayton CE, Marsh KA, Dyson A, et al. Ultrahigh-gradient acceleration of injected eletrons by laser-excited relativistic electron plasma waves. Phys Rev Lett. 1993;70:37-40. doi:10.1103/PhysRevLett.70.4034

7. Layland MK, Sessions DG, Lenox J. The influence of lymph node metastasis in the treatment of squamous cell carcinoma of the oral cavity, oropharynx, larynx, and hypopharynx: N0 versus $\mathrm{N}+$. Laryngoscope. 2005;115:629-639. doi:10.1097/01.mlg.0000161338. 54515.b1

8. Rosenthal EL, Warram JM, Bland KI, Zinn KR. The status of contemporary image-guided modalities in oncologic surgery. Ann Surg. 2015;261:46-55. doi:10.1097/SLA.0000000000000756
9. Van Driel PB, van de Giessen M, Boonstra MC, et al. Characterization and evaluation of the artemis camera for fluorescence-guided cancer surgery. Mol Imaging Biol MIB Off Publ Acad Mol Imaging. 2015;17:413-423. doi:10.1007/s11307-014-0799-z

10. Yokoyama J, Fujimaki M, Ohba S, et al. A feasibility study of NIR fluorescent image-guided surgery in head and neck cancer based on the assessment of optimum surgical time as revealed through dynamic imaging. OncoTargets Ther. 2013;6:325-330. doi:10.2147/OTT.S42006

11. Luo S, Zhang E, Su Y, Cheng T, Shi C. A review of NIR dyes in cancer targeting and imaging. Biomaterials. 2011;32:7127-7138. doi:10.1016/j.biomaterials.2011.06.024

12. Moore GE, Peyton WT. The clinical use of fluorescein in neurosurgery; the localization of brain tumors. $J$ Neurosurg. 1948;5:392-398. doi:10.3171/jns.1948.5.4.0392

13. Cho S, Salinas R, Lee J. Indocyanine-green for fluorescence-guided surgery of brain tumors: evidence, techniques, and practical experience. Front Surg. 2019;6:11. doi:10.3389/fsurg.2019.00011

14. Belykh E, Martirosyan NL, Yagmurlu K, et al. intraoperative fluorescence imaging for personalized brain tumor resection: current state and future directions. Front Surg. 2016;17(3):55.

15. Frangioni JV. New technologies for human cancer imaging. J Clin Oncol Off J Am Soc Clin Oncol. 2008;26:4012-4021. doi:10.1200/ JCO.2007.14.3065

16. Schaafsma BE, Mieog JSD, Hutteman M, et al. The clinical use of indocyanine green as a near-infrared fluorescent contrast agent for image-guided oncologic surgery. J Surg Oncol. 2011;104:323-332. doi:10.1002/jso.21943

17. Frangioni JV. The problem is background, not signal. Mol Imaging. 2009;8:303-304. doi:10.2310/7290.2009.00033

18. Lee JH, Park G, Hong GH, Choi J, Choi HS. Design considerations for targeted optical contrast agents. Quant Imaging Med Surg. 2012;2:266-273.

19. Jo D, Hyun H. Structure-inherent targeting of near-infrared fluorophores for image-guided surgery. Chonnam Med J. 2017;53:95102. doi: $10.4068 / \mathrm{cmj} .2017 .53 .2 .95$

20. Bredell MG. Sentinel lymph node mapping by indocyanin green fluorescence imaging in oropharyngeal cancer - preliminary experience. Head Neck Oncol. 2010;2:31. doi:10.1186/1758-3284-2-31

21. Van der Vorst JR, Schaafsma BE, Verbeek FPR, et al. Nearinfrared fluorescence sentinel lymph node mapping of the oral cavity in head and neck cancer patients. Oral Oncol. 2013;49:1519. doi:10.1016/j.oraloncology.2012.11.010

22. Atallah I, Milet C, Quatre R, et al. Role of near-infrared fluorescence imaging in the resection of metastatic lymph nodes in an optimized orthotopic animal model of HNSCC. Eur Ann Otorhinolaryngol Head Neck Dis. 2015;132:337-342. doi:10.10 16/j.anorl.2015.08.022

23. Digonnet A, van Kerckhove S, Moreau M, et al. Near infrared fluorescent imaging after intravenous injection of indocyanine green during neck dissection in patients with head and neck cancer: a feasibility study. Head Neck. 2016;38 Suppl 1:E1833E1837. doi:10.1002/hed.24331

24. Matsui A, Tanaka E, Choi HS, et al. Real-time intra-operative near-infrared fluorescence identification of the extrahepatic bile ducts using clinically available contrast agents. Surgery. 2010;148:87-95. doi:10.1016/j.surg.2009.12.004

25. Zelken JA, Tufaro AP. Current trends and emerging future of indocyanine green usage in surgery and oncology: an update. Ann Surg Oncol. 2015;22 Suppl 3:S1271-S1283. doi:10.1245/ s10434-015-4743-5

26. Moody ED, Viskari PJ, Colyer CL. Non-covalent labeling of human serum albumin with indocyanine green: a study by capillary electrophoresis with diode laser-induced fluorescence detection. J Chromatogr B Biomed Sci App. 1999;729:55-64. doi:10.1016/S0378-4347(99)00121-8 
27. Ogawa M, Kosaka N, Choyke PL, Kobayashi H. In vivo molecular imaging of cancer with a quenching near-infrared fluorescent probe using conjugates of monoclonal antibodies and indocyanine green. Cancer Res. 2009;69:1268-1272. doi:10.11 58/0008-5472.CAN-08-3660

28. Altinoğlu EI, Russin TJ, Kaiser JM, et al. Near-infrared emitting fluorophore-doped calcium phosphate nanoparticles for in vivo imaging of human breast cancer. ACS Nano. 2008;2:2075-2084. doi: $10.1021 / \mathrm{nn} 800448 \mathrm{r}$

29. Schulze T, Bembenek A, Schlag PM. Sentinel lymph node biopsy progress in surgical treatment of cancer. Langenbecks Arch Surg. 2004;389:532-550. doi:10.1007/s00423-004-0484-9

30. Owens EA, Henary M, El Fakhri G, Choi HS. Tissue-specific near-infrared fluorescence imaging. Acc Chem Res. 2016;49:1731-1740. doi:10.1021/acs.accounts.6b00239

31. Choi HS, Frangioni JV. Nanoparticles for biomedical imaging: fundamentals of clinical translation. Mol Imaging. 2010;9:291310. doi:10.2310/7290.2010.00031

32. Choi HS, Liu W, Liu F, et al. Design considerations for tumourtargeted nanoparticles. Nat Nanotechnol. 2010;5:42-47. doi:10.10 38/nnano.2009.314

33. Hellebust A, Richards-Kortum R. Advances in molecular imaging: targeted optical contrast agents for cancer diagnostics. Nanomed. 2012;7:429-445. doi:10.2217/nnm.12.12

34. Owens EA, Lee S, Choi J, Henary M, Choi HS. NIR fluorescent small molecules for intraoperative imaging. Wiley Interdiscip Rev Nanomed Nanobiotechnol. 2015;7:828-838. doi:10.1002/ wnan. 1337

35. Kobayashi H, Ogawa M, Alford R, Choyke PL, Urano Y. New strategies for fluorescent probe design in medical diagnostic imaging. Chem Rev. 2010;110:2620-2640. doi:10.1021/cr900263j

36. Faraji AH, Wipf P. Nanoparticles in cellular drug delivery. Bioorg Med Chem. 2009;17:2950-2962. doi:10.1016/j.bmc.2009.02.043

37. Moghimi SM, Szebeni J. Stealth liposomes and long circulating nanoparticles: critical issues in pharmacokinetics, opsonization and protein-binding properties. Prog Lipid Res. 2003;42:463478. doi:10.1016/S0163-7827(03)00033-X

38. Saxena V, Sadoqi M, Shao J. Degradation kinetics of indocyanine green in aqueous solution. J Pharm Sci. 2003;92:2090-2097. doi:10.1002/jps.10470

39. Mordon S, Devoisselle JM, Soulie-Begu S, Desmettre T. Indocyanine green: physicochemical factors affecting its fluorescence in vivo. Microvasc Res. 1998;55:146-152. doi:10.1006/ mvre.1997.2055

40. Wang H, Li X, Tse BW-C, et al. Indocyanine green-incorporating nanoparticles for cancer theranostics. Theranostics. 2018;8:12271242. doi:10.7150/thno. 22872

41. Fang J, Nakamura H, Maeda H. The EPR effect: unique features of tumor blood vessels for drug delivery, factors involved, and limitations and augmentation of the effect. Adv Drug Deliv Rev. 2011;63:136-151. doi:10.1016/j.addr.2010.04.009

42. Bertrand N, Wu J, Xu X, Kamaly N, Farokhzad OC. Cancer nanotechnology: the impact of passive and active targeting in the era of modern cancer biology. Adv Drug Deliv Rev. 2014;66:2-25. doi:10.1016/j.addr.2013.11.009

43. Millard M, Yakavets I, Zorin V, Kulmukhamedova A, Marchal S, Bezdetnaya L. Drug delivery to solid tumors: the predictive value of the multicellular tumor spheroid model for nanomedicine screening. Int J Nanomedicine. 2017;12:7993-8007. doi:10.21 47/IJN.S146927

44. Bergers G, Benjamin LE. Tumorigenesis and the angiogenic switch. Nat Rev Cancer. 2003;3:401-410. doi:10.1038/nrc1093

45. Hobbs SK, Monsky WL, Yuan F, et al. Regulation of transport pathways in tumor vessels: role of tumor type and microenvironment. Proc Natl Acad Sci U S A. 1998;95:4607-4612. doi:10.10 73/pnas.95.8.4607
46. Hashizume H, Baluk P, Morikawa S, et al. Openings between defective endothelial cells explain tumor vessel leakiness. Am J Pathol. 2000;156:1363-1380. doi:10.1016/S0002-9440(10)64698-6

47. Iyer AK, Khaled G, Fang J, Maeda H. Exploiting the enhanced permeability and retention effect for tumor targeting. Drug Discov Today. 2006;11:812-818. doi:10.1016/j.drudis.2006.07. 005

48. Jang SH, Wientjes MG, Lu D, Au JLS. Drug delivery and transport to solid tumors. Pharm Res. 2003;20:1337-1350. doi:10.10 23/A:1025785505977

49. Lee C-H, Cheng S-H, Wang Y-J, et al. Near-infrared mesoporous silica nanoparticles for optical imaging: characterization and in vivo biodistribution. Adv Funct Mater. 2009;19: 215-222. doi:10.1002/adfm.200800753

50. Trewyn BG, Giri S, Slowing II, Lin VS-Y. Mesoporous silica nanoparticle based controlled release, drug delivery, and biosensor systems. Chem Commun Camb Engl. 2007;3236-3245. doi:10.1039/b701744h

51. Souris JS, Lee C-H, Cheng S-H, et al. Surface charge-mediated rapid hepatobiliary excretion of mesoporous silica nanoparticles. Biomaterials. 2010;31:5564-5574. doi:10.1016/j.biomaterials.2010. 01.042

52. Chen J, Liu C, Zeng G, et al. Indocyanine green loaded reduced graphene oxide for in vivo photoacoustic/fluorescence dual-modality tumor imaging. Nanoscale Res Lett. 2016;11:85. doi:10.1186/s11671-016-1288-x

53. Ashokan A, Gowd GS, Somasundaram VH, et al. Multifunctional calcium phosphate nano-contrast agent for combined nuclear, magnetic and near-infrared in vivo imaging. Biomaterials. 2013;34:7143-7157. doi:10.1016/j.biomaterials.2013.05.077

54. Song $\mathrm{S}$, Shen $\mathrm{H}$, Yang $\mathrm{T}$, et al. Indocyanine green loaded magnetic carbon nanoparticles for near infrared fluorescence/magnetic resonance dual-modal imaging and photothermal therapy of tumor. ACS Appl Mater Interfaces. 2017;9:9484-9495. doi:10.1021/acsami.7b00490

55. Zhao $\mathrm{P}$, Zheng $\mathrm{M}$, Yue $\mathrm{C}$, et al. Improving drug accumulation and photothermal efficacy in tumor depending on size of ICG loaded lipid-polymer nanoparticles. Biomaterials. 2014;35:6037-6046. doi:10.1016/j.biomaterials.2014.01.026

56. Wu L, Fang S, Shi S, Deng J, Liu B, Cai L. Hybrid polypeptide micelles loading indocyanine green for tumor imaging and photothermal effect study. Biomacromolecules. 2013;14:3027-3033. doi:10.1021/bm400426f

57. Kim TH, Chen Y, Mount CW, Gombotz WR, Li X, Pun SH. Evaluation of temperature-sensitive, indocyanine green-encapsulating micelles for noninvasive near-infrared tumor imaging. Pharm Res. 2010;27:1900-1913. doi:10.1007/s11095-010-0190-y

58. Mok H, Jeong H, Kim S-J, Chung BH. Indocyanine green encapsulated nanogels for hyaluronidase activatable and selective near infrared imaging of tumors and lymph nodes. Chem Commun Camb Engl. 2012;48:8628-8630. doi:10.1039/c2cc33555g

59. Hill TK, Abdulahad A, Kelkar SS, et al. Indocyanine greenloaded nanoparticles for image-guided tumor surgery. Bioconjug Chem. 2015;26:294-303. doi:10.1021/bc5005679

60. Luk BT, Fang RH, Zhang L. Lipid- and polymer-based nanostructures for cancer theranostics. Theranostics. 2012;2:11171126. doi: $10.7150 /$ thno. 4381

61. Suganami A, Iwadate Y, Shibata S, et al. Liposomally formulated phospholipid-conjugated indocyanine green for intra-operative brain tumor detection and resection. Int $J$ Pharm. 2015;496:401-406. doi:10.1016/j.ijpharm.2015.10.001

62. Mérian J, Boisgard R, Bayle P-A, Bardet M, Tavitian B, Texier I. Comparative biodistribution in mice of cyanine dyes loaded in lipid nanoparticles. Eur J Pharm Biopharm Off J Arbeitsgemeinschaft Pharm Verfahrenstechnik EV. 2015;93:1-10. doi:10.1016/j. ejpb.2015.03.019 
63. Chopra A. Folic acid-indocyanine green-poly(d,1-lactide-coglycolide)-lipid nanoparticles. In: Molecular Imaging and Contrast Agent Database (MICAD) [internet]. Bethesda (MD): National Center for Biotechnology Information (US); 2004.

64. Xin Y, Liu T, Yang C. Development of PLGA-lipid nanoparticles with covalently conjugated indocyanine green as a versatile nanoplatform for tumor-targeted imaging and drug delivery. Int $J$ Nanomedicine. 2016;11:5807-5821. doi:10.2147/IJN.S119999

65. Zheng $\mathrm{C}$, Zheng $\mathrm{M}$, Gong $\mathrm{P}$, et al. Indocyanine green-loaded biodegradable tumor targeting nanoprobes for in vitro and in vivo imaging. Biomaterials. 2012;33:5603-5609. doi:10.1016/j. biomaterials.2012.04.044

66. Zheng X, Xing D, Zhou F, Wu B, Chen WR. Indocyanine greencontaining nanostructure as near infrared dual-functional targeting probes for optical imaging and photothermal therapy. Mol Pharm. 2011;8:447-456. doi:10.1021/mp200125j

67. Wu $\mathrm{H}$, Wang $\mathrm{H}$, Liao $\mathrm{H}$, et al. Multifunctional nanostructures for tumor-targeted molecular imaging and photodynamic therapy. Adv Healthc Mater. 2016;5:311-318. doi:10.1002/adhm.2015006 68

68. Yan L, Qiu L. Indocyanine green targeted micelles with improved stability for near-infrared image-guided photothermal tumor therapy. Nanomed. 2015;10:361-373. doi:10.2217/nnm.14.118

69. Rodriguez VB, Henry SM, Hoffman AS, Stayton PS, Li X, Pun $\mathrm{SH}$. Encapsulation and stabilization of indocyanine green within poly(styrene-alt-maleic anhydride) block-poly(styrene) micelles for near-infrared imaging. $J$ Biomed Opt. 2008;13:14025. doi: $10.1117 / 1.2834296$

70. Uthaman S, Bom J, Kim HS, et al. Tumor homing indocyanine green encapsulated micelles for near infrared and photoacoustic imaging of tumors. J Biomed Mater Res B Appl Biomater. 2016;104:825-834. doi:10.1002/jbm.b.33607

71. Qi B, Crawford AJ, Wojtynek NE, et al. Indocyanine green loaded hyaluronan-derived nanoparticles for fluorescenceenhanced surgical imaging of pancreatic cancer. Nanomed Nanotechnol Biol Med. 2018;14:769-780. doi:10.1016/j.nano. 2017.12.015

72. Dosio F, Arpicco S, Stella B, Fattal E. Hyaluronic acid for anticancer drug and nucleic acid delivery. Adv Drug Deliv Rev. 2016;97:204-236. doi:10.1016/j.addr.2015.11.011

73. Bahmani B, Guerrero Y, Bacon D, Kundra V, Vullev VI, Anvari B. Functionalized polymeric nanoparticles loaded with indocyanine green as theranostic materials for targeted molecular near infrared fluorescence imaging and photothermal destruction of ovarian cancer cells. Lasers Surg Med. 2014;46:582-592. doi:10.1002/lsm.v46.7

74. Mac JT, Nuñez V, Burns JM, Guerrero YA, Vullev VI, Anvari B. Erythrocyte-derived nano-probes functionalized with antibodies for targeted near infrared fluorescence imaging of cancer cells. Biomed Opt Express. 2016;7:1311-1322. doi:10.1364/BOE.7.00 1311

75. Yamaguchi H, Tsuchimochi M, Hayama K, Kawase T, Tsubokawa N. Dual-labeled near-infrared/(99m)Tc imaging probes using PAMAM-coated silica nanoparticles for the imaging of HER2-expressing cancer cells. Int J Mol Sci. 2016;17(7): E1086. doi:10.3390/ijms17071086.

76. Barthélémy P, Leblanc J, Goldbarg V, Wendling F, Kurtz J-E. Pertuzumab: development beyond breast cancer. Anticancer Res. 2014;34:1483-1491.

77. Steffensen KD, Waldstrøm M, Andersen RF, et al. Protein levels and gene expressions of the epidermal growth factor receptors, HER1, HER2, HER3 and HER4 in benign and malignant ovarian tumors. Int J Oncol. 2008;33:195-204.

78. Chen W, Ayala-Orozco C, Biswal NC, et al. Targeting pancreatic cancer with magneto-fluorescent theranostic gold nanoshells. Nanomed. 2014;9:1209-1222. doi:10.2217/nnm.13.84
79. Kolitz-Domb M, Grinberg I, Corem-Salkmon E, Margel S Engineering of near infrared fluorescent proteinoid-poly(L-lactic acid) particles for in vivo colon cancer detection. $J$ Nanobiotechnology. 2014;12:30. doi:10.1186/s12951-014-0030-z

80. Patel RH, Wadajkar AS, Patel NL, Kavuri VC, Nguyen KT, Liu H. Multifunctionality of indocyanine green-loaded biodegradable nanoparticles for enhanced optical imaging and hyperthermia intervention of cancer. $J$ Biomed Opt. 2012;17:46003. doi:10.1117/1.JBO.17.4.046003

81. Ding J, Feng M, Wang F, Wang H, Guan W. Targeting effect of PEGylated liposomes modified with the Arg-Gly-Asp sequence on gastric cancer. Oncol Rep. 2015;34:1825-1834. doi:10.3892/ or. 2015.4142

82. Chen Z, Zhao P, Luo Z, et al. Cancer cell membrane-biomimetic nanoparticles for homologous-targeting dual-modal imaging and photothermal therapy. ACS Nano. 2016;10(11):10049-10057. doi:10.1021/acsnano.6b04695

83. Kovalska VB, Volkova KD, Losytskyy MY, Tolmachev OI, Balanda AO, Yarmoluk SM. 6,6'-Disubstituted benzothiazole trimethine cyanines-new fluorescent dyes for DNA detection. Spectrochim Acta A Mol Biomol Spectrosc. 2006;65:271-277. doi:10.1016/j.saa.2005.10.042

84. Yarmoluk SM, Kovalska VB, Lukashov SS, Slominskii YL. Interaction of cyanine dyes with nucleic acids. XII.beta-substituted carbocyanines as possible fluorescent probes for nucleic acids detection. Bioorg Med Chem Lett. 1999;9:1677-1678. doi:10.1016/S0960-894X(99)00253-X

85. Nakajima T, Mitsunaga M, Bander NH, Heston WD, Choyke PL, Kobayashi H. Targeted, activatable, in vivo fluorescence imaging of prostate-specific membrane antigen (PSMA) positive tumors using the quenched humanized J591 antibody-indocyanine green (ICG) conjugate. Bioconjug Chem. 2011;22:1700-1705. doi:10.1021/bc2002715

86. Sano K, Nakajima T, Miyazaki K, et al. Short PEG-linkers improve the performance of targeted, activatable monoclonal antibody-indocyanine green optical imaging probes. Bioconjug Chem. 2013;24:811-816. doi:10.1021/bc400050k

87. Villaraza AJL, Milenic DE, Brechbiel MW. Improved speciation characteristics of PEGylated indocyanine green-labeled Panitumumab: revisiting the solution and spectroscopic properties of a near-infrared emitting anti-HER1 antibody for optical imaging of cancer. Bioconjug Chem. 2010;21:2305-2312. doi:10.10 21/bc100336b

88. Zhou Y, Kim Y-S, Milenic DE, Baidoo KE, Brechbiel MW. In vitro and in vivo analysis of indocyanine green-labeled panitumumab for optical imaging-a cautionary tale. Bioconjug Chem. 2014;25:1801-1810. doi:10.1021/bc500312w

89. Zalipsky S. Functionalized poly(ethylene glycol) for preparation of biologically relevant conjugates. Bioconjug Chem. 1995;6:150-165. doi:10.1021/bc00032a002

90. Johnson AJ, Karpatkin MH, Newman J. [Clinical investigation of intermediate- and high-purity antihaemophilic factor (factor VIII) concentrates]. Br J Haematol. 1971;21:21-41. doi:10.1111/ j.1365-2141.1971.tb03414.x

91. Mujumdar RB, Ernst LA, Mujumdar SR, Waggoner AS. Cyanine dye labeling reagents containing isothiocyanate groups. Cytometry. 1989;10:11-19. doi:10.1002/(ISSN)1097-0320

92. Sano K, Ohashi M, Kanazaki K, et al. In vivo photoacoustic imaging of cancer using indocyanine green-labeled monoclonal antibody targeting the epidermal growth factor receptor. Biochem Biophys Res Commun. 2015;464:820-825. doi:10.1016/j.bbrc. 2015.06.157

93. Zhang C, Zhang Y, Hong K, Zhu S, Wan J. Photoacoustic and fluorescence imaging of cutaneous squamous cell carcinoma in living subjects using a probe targeting integrin $\alpha v \beta 6$. Sci Rep. 2017;7:42442. doi:10.1038/srep42442 
94. Janes SM, Watt FM. Switch from alphavbeta5 to alphavbeta6 integrin expression protects squamous cell carcinomas from anoikis. J Cell Biol. 2004;166:419-431. doi:10.1083/jcb.200312074

95. Van Aarsen LAK, Leone DR, Ho S, et al. Antibody-mediated blockade of integrin alpha $\mathrm{v}$ beta 6 inhibits tumor progression in vivo by a transforming growth factor-beta-regulated mechanism. Cancer Res. 2008;68:561-570. doi:10.1158/0008-5472.CAN-072307

96. Sano K, Nakajima T, Ali T, et al. Activatable fluorescent cysdiabody conjugated with indocyanine green derivative: consideration of fluorescent catabolite kinetics on molecular imaging. $J$ Biomed Opt. 2013;18:101304. doi:10.1117/1.JBO.18.10.101304

97. Baik FM, Hansen S, Knoblaugh SE, et al. fluorescence identification of head and neck squamous cell carcinoma and high-risk oral dysplasia with BLZ-100, a chlorotoxin-indocyanine green conjugate. JAMA Otolaryngol-Head Neck Surg. 2016;142:330-338. doi:10.1001/jamaoto.2015.3617

98. Soroceanu L, Gillespie Y, Khazaeli MB, Sontheimer H. Use of chlorotoxin for targeting of primary brain tumors. Cancer Res. 1998;58:4871-4879.

99. Veiseh M, Gabikian P, Bahrami S-B, et al. Tumor paint: a chlorotoxin: cy5.5bioconjugate for intraoperative visualization of cancer foci. Cancer Res. 2007;67:6882-6888. doi:10.1158/0008-5472. CAN-06-3948

100. Stroud MR, Hansen SJ, Olson JM. In vivo bio-imaging using chlorotoxin-based conjugates. Curr Pharm Des. 2011;17:43624371. doi:10.2174/138161211798999375
101. Kesavan K, Ratliff J, Johnson EW, et al. Annexin A2 is a molecular target for TM601, a peptide with tumor-targeting and anti-angiogenic effects. $J$ Biol Chem. 2010;285:4366-4374. doi:10.1074/jbc.M109.066092

102. Deshane J, Garner CC, Sontheimer H. Chlorotoxin inhibits glioma cell invasion via matrix metalloproteinase-2. J Biol Chem. 2003;278:4135-4144. doi:10.1074/jbc.M205662200

103. Zhang C, Zhou L, Zhang J, et al. Green and facile synthesis of a theranostic nanoprobe with intrinsic biosafety and targeting abilities. Nanoscale. 2016;8:16204-16211. doi:10.1039/C6NR018 $45 \mathrm{~A}$

104. Belykh E, Martirosyan NL, Yagmurlu K, et al. Intraoperative fluorescence imaging for personalized brain tumor resection: current state and future directions. Front Surg. 2016;3:55. doi:10.3 389/fsurg.2016.00055

105. Antaris AL, Chen H, Cheng K, et al. A small-molecule dye for NIR-II imaging. Nat Mater. 2016;15:235-242. doi:10.1038/ nmat4476

106. Bhavane R, Starosolski Z, Stupin I, Ghaghada KB, Annapragada A. NIR-II fluorescence imaging using indocyanine green nanoparticles. Sci Rep. 2018;8:14455. doi:10.1038/s41598-018-32754-y

107. Zeh R, Sheikh S, Xia L, et al. The second window ICG technique demonstrates a broad plateau period for near infrared fluorescence tumor contrast in glioblastoma. PLoS One. 2017;12:e0182034. doi:10.1371/journal.pone.0182034
International Journal of Nanomedicine

\section{Publish your work in this journal}

The International Journal of Nanomedicine is an international, peerreviewed journal focusing on the application of nanotechnology in diagnostics, therapeutics, and drug delivery systems throughout the biomedical field. This journal is indexed on PubMed Central, MedLine, CAS, SciSearch ${ }^{\mathbb{}}$, Current Contents ${ }^{\mathbb{R}} /$ Clinical Medicine,

\section{Dovepress}

Journal Citation Reports/Science Edition, EMBase, Scopus and the Elsevier Bibliographic databases. The manuscript management system is completely online and includes a very quick and fair peer-review system, which is all easy to use. Visit http://www.dovepress.com/ testimonials.php to read real quotes from published authors. 